\title{
Erratum to: Dark matter direct detection of a fermionic singlet at one loop
}

\author{
Juan Herrero-García ${ }^{1, a}$, Emiliano Molinaro ${ }^{2, b}$, Michael A. Schmidt ${ }^{3, c}$ \\ ${ }^{1}$ Departamento de Fìsica Teòrica and IFIC, Universidad de Valencia-CSIC, C/Catedràtico Josè Beltràn, 2, 46980 Paterna, Spain \\ 2 Department of Mathematics and Computer Science (IMADA), University of Southern Denmark, Campusvej 55, 5230 Odense M, Denmark \\ ${ }^{3}$ Sydney Consortium for Particle Physics and Cosmology, School of Physics, The University of New South Wales, New South Wales 2052 , Australia
}

Received: 21 December 2021 / Accepted: 6 January 2022 / Published online: 19 January 2022

(C) The Author(s) 2022

\begin{abstract}
This erratum corrects Fig. 3a in [1] and clarifies the enhanced direct detection rate of the Dirac dark matter scenario with a vector-like fermion.
\end{abstract}

\section{Erratum to: Eur. Phys. J. C https://doi.org/10.1140/epjc/s10052-018-5935-5}

The complex phase of the Yukawa coupling $y_{A}$ has been erroneously set to zero in the code for Fig. 3a in [1]. The corrected figure is shown in Fig. 1. The Wilson coefficients $c_{1}^{N}$ change sign at about $m_{\psi} \simeq 225 \mathrm{GeV}$ and $c_{4,5,6}^{N}$ at $m_{\psi} \simeq$ $425 \mathrm{GeV}$. In the Majorana dark matter (DM) case, all the Wilson coefficients are in the range $10^{-5}-10^{-4}$, except in the region of DM mass when a Wilson coefficient changes sign. No other results have been affected by this error.
We further want to clarify that the large enhancement of the differential rate for Dirac DM with a vector-like fermion in Fig. 4a in [1] is mainly due to the electric dipole moment $d_{\psi}$. The contribution from the magnetic dipole moment $\mu_{\psi}$ is large but subdominant, because the relevant non-relativistic operator $\mathcal{O}_{5}^{N}$ is suppressed by $\vec{v}_{\perp}$ compared to $\mathcal{O}_{11}^{N}$. The different spectral shapes in Fig. $4 \mathrm{~b}$ in [1] are due to the absence of dipole moments for Majorana DM.

The original article can be found online at https://doi.org/10.1140/ epjc/s10052-018-5935-5.

\footnotetext{
a e-mail: juan.herrero@ific.uv.es

b e-mail: molinaro@imada.sdu.dk

c e-mail: m.schmidt@unsw.edu.au (corresponding author)
} 


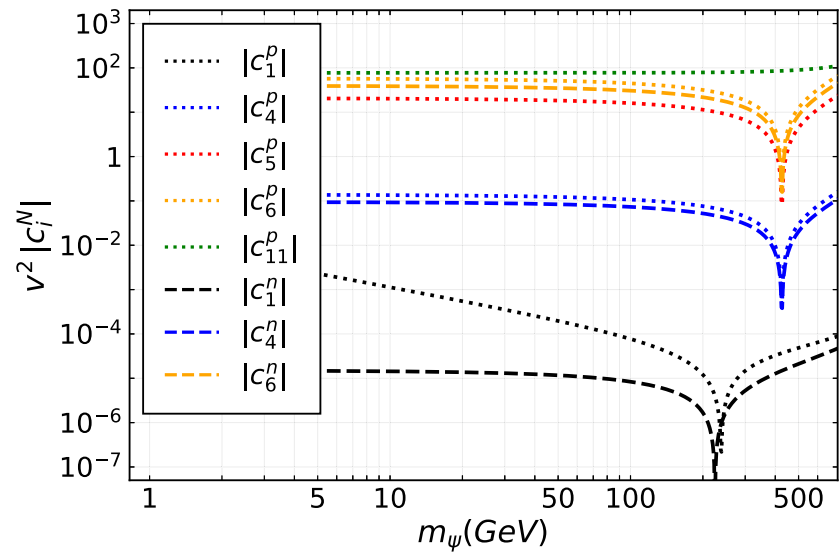

Fig. 1 Dirac DM (top) and Majorana DM (bottom) with a vectorlike fermion $F$ of mass $m_{F}=600 \mathrm{GeV}$ and a scalar $S$ of mass $m_{S}=500 \mathrm{GeV}$. The Yukawa couplings are fixed to $y_{V}=1$ and $y_{A}=1.3 e^{i 1.4}$ and Higgs portal coupling $\lambda_{H S}=3$. The Wilson coef-

Acknowledgements We would like to thank Michael Gustafsson for pointing out the error.

Open Access This article is licensed under a Creative Commons Attribution 4.0 International License, which permits use, sharing, adaptation, distribution and reproduction in any medium or format, as long as you give appropriate credit to the original author(s) and the source, provide a link to the Creative Commons licence, and indicate if changes were made. The images or other third party material in this article are included in the article's Creative Commons licence, unless indicated otherwise in a credit line to the material. If material is not included in the article's Creative Commons licence and your intended use is not permitted by statutory regulation or exceeds the permitted use, you will need to obtain permission directly from the copyright holder. To view a copy of this licence, visit http://creativecomm ons.org/licenses/by/4.0/.

Funded by SCOAP ${ }^{3}$.

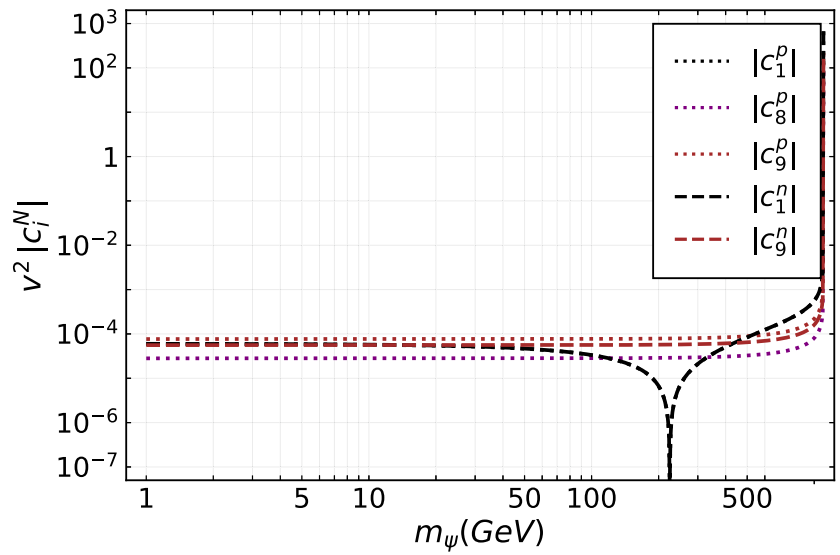

ficients are evaluated for ${ }_{54}^{132} \mathrm{Xe}$ at $E_{R}=8.59 \mathrm{keV}$ and are displayed in dimensionless units by rescaling with the square of the electroweak $\operatorname{VEV} v=246.2 \mathrm{GeV}$

\section{Reference}

1. J. Herrero-Garcia, E. Molinaro, M.A. Schmidt, Eur. Phys. J. C 78(6), 471 (2018). https://doi.org/10.1140/epjc/s10052-018-5935-5 